\title{
IMP3 is a novel prognostic marker for colon cancer
}

Colon cancer is one of the leading causes of cancer-related mortality. Previous studies using laser capture microdissection revealed insulin-like growth factor-II mRNA-binding protein 3 (IMP3) was overexpressed in colon cancer compared with normal mucosa, indicating its role as a candidate biomarker.

Therefore, Li and coauthors assessed IMP3 expression by tissue microarray analysis in 203 patients with primary colon tumors, normal mucosa or lymphnode metastases. "This is the first study to highlight the clinical significance of IMP3 in colon cancer" comment the researchers.

Immunostaining revealed that IMP3 was significantly higher in patients with lymph-node metastases (91\%) than primary colon cancer $(65 \%)$ or normal mucosa (3.9\%). IMP3 levels were higher in colon cancer samples compared with normal colon samples. Increased IMP3 expression was significantly associated with a higher tumor stage, lymph-node metastases, distant metastases and overexpression of Ki-67. Importantly, patients with elevated IMP3 levels had more-aggressive tumors with an almost 11-fold increased risk of distant metastases compared with IMP3-negative tumors. Those with IMP3-positive tumors had a significantly lower 5-year disease-free survival and overall survival. Moreover, multivariate analysis revealed IMP3 was an independent prognostic marker for disease-free survival.

"Our study indicates that IMP3 might play a critical role in the proliferation and progression of colon cancer and could serve as a novel biomarker to identify patients at risk of developing metastasis" said Zhihai Peng, lead investigator.

\section{Lisa Hutchinson}

Original article Li, D. et al. IMP3 is a novel prognostic marker that correlates with colon cancer progression and pathogenesis. Ann. Surg. Oncol. 16, 3499-3506 (2009) 